\title{
Electron Paramagnetic Resonance Study of Organic Radicals in Some Herbal Plants
}

\author{
A. Krupska ${ }^{a}$, E. Wojtowicz ${ }^{b}$ And M.A. Augustyniak-JabŁOKOW ${ }^{a}$ \\ ${ }^{a}$ Institute of Molecular Physics, Polish Academy of Sciences, Poznań, Poland \\ ${ }^{b}$ Department of Food Concentrates and Starch Products, \\ Prof. Wacław Dąbrowski Institute of Agricultural and Food Biotechnology, Poznań, Poland
}

(Received January 18, 2016; in final form May 1, 2016)

\begin{abstract}
In this work we show the impact of the roasting process on the properties of organic radicals in some herbal products added to cereal coffee. The EPR study shows the presence of radicals in dried herbal products, both natural and roasted. Changes in the relative intensity of the EPR signal and in the $g$-factor for unroasted and roasted plant material can be observed. This indicates to the recombination processes of radicals. Roasting process increases the number of radicals. The analysis of EPR spectra show that they consist of two Gaussian lines which indicate that there are at least two different kinds of radicals. The values of $g$-factor about 2 may be derived from a number of very different radicals. For this reason the identification of such radicals is not strictly possible.
\end{abstract}

DOI: 10.12693/APhysPolA.129.1201

PACS/topics: 76.30.-v, 76.30.Rn

\section{Introduction}

Roasted plant products, in particular herbs, are often added to various foods, e.g. cereal coffee. They give a specific aroma and flavor of the products. Researchers are constantly looking for new interesting materials which by its specific composition will be more attractive aroma of cereal coffee [1] and even zoom into the aroma of natural coffee [2]. Selection of herbal raw materials as new additives for grain coffee is preferred because of their bioactive components. Herbs are an important element in the prevention and treatment of diseases, and nutrition. The main factor is the effectiveness of the health-enhancing antioxidant capacity that results from the mechanism of action of bioactive compounds, including polyphenols. Prior to addition to the cereal coffee these raw materials are subjected to a roasting process, which may similarly as in the case of coffee $[3,4]$ to generate free radicals pro-oxidative properties.

Observed in the plant radicals are long-lived radicals. Long-lived radicals that can be observed directly without the use of spin traps are not reactive. These the long-lived radicals can be generated thermally, mechanochemically, by ionizing radiation.

Formation of long-lived free radicals from acylpyridinium salts with alkali was studied by EPR, NMR, UV absorption and visible absorption [5]. Long-lived free radicals formed during the reaction of $\mathrm{H}_{2} \mathrm{O}_{2}$ and metmyoglobin in the presence of bovine serum albumin (BSA) were investigated using freeze quench and spin-trap ESR spectroscopy [6]. Long-lived organic radicals were studied by EPR in the carp liver [7]. The EPR study shows more than 10 EPR signals from different radicals. Free radicals with a long life time in the leaves of two rice cultivars were studied by EPR [8]. Authors analyzed the yield of long-lived radicals in leaves of Sasanishiki and Norin-1 grown under UVB irradiation. Authors suppose that observed radicals may be attributable to $P_{700}$ cation radicals in the reaction center of photosystem I, and tyrosine cation radicals in the reaction center of photosystem II, respectively. It was proved that thermally generated starch radicals are very stable $[9,10]$. Thermally generated free radicals (in $160^{\circ} \mathrm{C}$ ) in the drug drotaverine were examined by EPR to check whether the use of heat sterilization is justified [11]. EPR spectra of unirradiated and 743 Gy gamma irradiated bovine Hb samples were recorded at room temperature [12]. Long-lived radical cations were used as a model compounds for the reactive one-electron oxidation product of vitamin $\mathrm{E}$ [13]. A new type of radiation induced radicals with a long life-time in cells were observed using EPR spectroscopy [14]. Authors suppose that these type radicals are probably cause of mutations and morphological transformation in cells. $\mathrm{NO} /$ air/olefin may be one of mechanism responsible for the produced long-lived radicals in smoke [15]. Longlived albumin-derived radicals obtained by incubation of HRP with $\mathrm{H}_{2} \mathrm{O}_{2}$ and bovine or human serum albumins, in the presence and absence of tyrosine was detected by EPR spectroscopy [16].

EPR studies for two samples of green coffee beans were performed [3]. EPR studies were carried out as a function of temperature and time. With increase of the roasting temperature and time (16 min maximally) the intensity of the EPR signal varies and the $g$-factor decreases. Authors claim that the radicals produced on roasting are chemically distinct from those in the green beans. This means that we are dealing with two various radicals. But the authors do not specify what the radicals are. Arabica and Columbia coffee roasted in air and $\mathrm{N}_{2}$ were also studied by EPR [4]. The atmosphere air and $\mathrm{N}_{2}$ were used in the roasting and cooling process. EPR spectra show that the free radical contents increased with increasing roasting time. Carbon centered and oxygen centered 
radicals were detected by EPR in the asparagus root and stem [17]. Effect of time of $\gamma$-irradiation in decay in longlived organic radicals from hamster embryo and effect of vitamin $\mathrm{C}$ on the yields of these radicals were observed by EPR spectroscopy [18].

In this work we show the impact of the roasting process on the properties of organic radicals in some herbal products added to cereal coffee. We use EPR techniques to our investigation.

\section{Materials and methods}

The experimental material was plant products, mainly herbs both natural and roasted. It was in the form of powder. Dried novel raw materials: artichoke, sea buckthorn fruits, dandelion roots, lovage roots, hawthorn fruits, rose hips, rowanberry were purchased from retail outlets. All materials were roasted. Samples before and after roasting were ground using a W Z1 laboratory mill (ZBPP, Bydgoszcz, Poland) and roasted in a BRZ 2/4/6 sample roaster battery (PROBAT, Emmerich am Rhein, Germany). Samples (100 g) were roasted in $160^{\circ} \mathrm{C}$ for $8 \mathrm{~min}$ to obtain dark roasts.

The samples were investigated at room temperatures with an EPR spectrometer (RADIOPAN, Poznań, Poland), in a standard tube, in the X-band $(9.4 \mathrm{GHz})$ with magnetic field modulation of $100 \mathrm{kHz}$. Ruby crystal served as a standard of the EPR relative intensity.

\section{Results and interpretation}

\subsection{Results}

The presented results were obtained for dried unroasted and roasted artichoke, sea buckthorn fruits, dandelion roots, lovage roots, hawthorn fruits, rose hips, rowanberry. Figure 1 shows an example of changes in the EPR spectra for unroasted and roasted artichoke and dandelion roots. In these plant materials the EPR signal was the best visible. It can be seen shift to the left of the EPR line of the roasted material in comparison to the unroasted one. It is demonstrated in the $g$ parameter increases for roasted material. These values are summarized in Table I. Table I also shows the difference

TABLE I

Changes in the average $g$-factors and relative intensity $I_{\text {rel }}$ for unroasted and roasted materials.

\begin{tabular}{c|c|c|c|c}
\hline $\begin{array}{c}\text { Dried plant } \\
\text { material }\end{array}$ & $\begin{array}{c}g_{\mathrm{av}} \\
\text { for unroasted } \\
\text { (from fitting) }\end{array}$ & $\begin{array}{c}g_{\mathrm{av}} \\
\text { for roasted } \\
\text { (from fitting) }\end{array}$ & $\begin{array}{c}I_{\text {rel }} \text { for } \\
\text { unroasted }\end{array}$ & $\begin{array}{c}I_{\text {rel }} \text { for } \\
\text { roasted }\end{array}$ \\
\hline $\begin{array}{c}\text { artichoke } \\
\text { dandelion root } \\
\text { lovage root }\end{array}$ & 2.00239 & 2.00298 & 0.037 & 0.8 \\
sea buckthorn & 2.00168 & 2.0038 & 0.0325 & 0.45 \\
fruit & 2.00162 & 2.00292 & 0.024 & 0.59 \\
hawthorn fruit & - & - & 0.005 & 0.62 \\
rose hip & - & - & 0.031 & 0.4 \\
rowanberry & - & - & 0.042 & 0.2
\end{tabular}

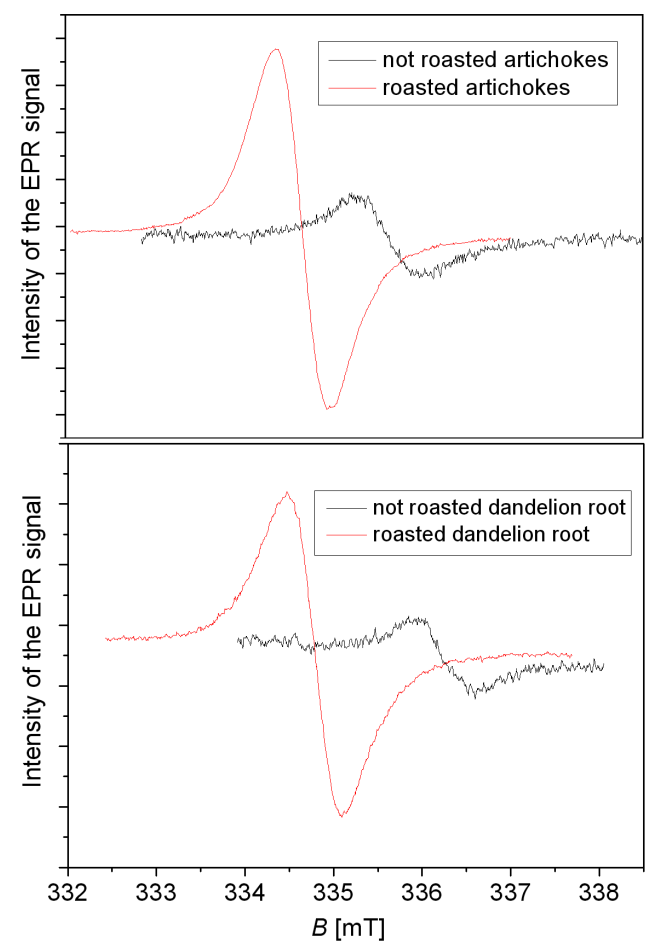

Fig. 1. An example of the EPR spectrum obtained for the non-roasted and roasted artichoke (upper graph) and dandelion root (bottom graph).

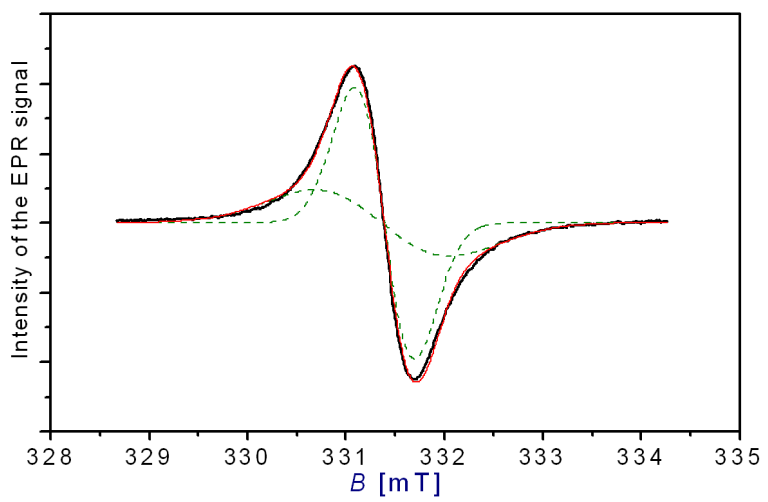

Fig. 2. The example of fitting for two Gaussian lines for roasted artichoke. Continuous red line shows a fit of two Gaussian lines. The dotted green lines show two components of EPR spectra. $B$ denotes the magnetic field.

in relative intensity $I_{\text {rel }}$ of roasted and unroasted material. The relative intensity of EPR signal was calculated by comparison of the line intensity of the sample being under investigation and the line intensity of the ruby crystal. The absolute values of the EPR parameters and their accuracy were calculated by using a computer program. The analysis of the EPR spectra was performed by computer simulation assuming two Gaussian lines. EPR spectra could not to fit in single Gaussian or single Lorentzian line. A good fit was obtained for two 
Gaussian lines. Figure 2 shows an example of fitting for two Gaussian lines for roasted artichoke. The Lorentz fit to the experimental EPR line is not justified, because of lack of conduction electrons and inter-chainable interactions. As seen from the Table I $g$ factor (obtained from the fitting procedure for some samples) is different for unroasted and roasted plant materials. Changes in the relative intensity of EPR signal for unroasted and roasted dried samples are also visible.

\subsection{Results interpretation}

- The differences in the relative intensity of EPR signals of unroasted and roasted material indicate on the amount of radicals.

- The shift of the EPR signal for the roasted material regarding for the unroasted one may indicate the occurrence of certain processes of recombination of radicals under the influence of roasting.

- The presence of two components of EPR spectra indicates that there are at least two different radicals.

- In the case of complex biological materials EPR signal $g \approx 2$ may be derived from a number of very different radicals. The identification of such radicals is not strictly possible. We can only guess what kind may be radicals. We suppose that our radicals can be derived from an organic iron compound. However this is a hypothesis.

\section{Discussion}

The EPR study shows the presence of radicals in dried herbal products, both natural and roasted. As indicated by the relative intensity of the EPR signal, roasting of the product increases significantly the number of radicals. This is probably due to detachment of electrons and/or its faster flow. It is possible that the drying process could already generate radicals. Drying may affect the formation of specific radicals. The results indicate that we have more than one type of radical. Presumably, there are several different radicals in our material. We have a mixture of different radicals with $g$-factor about 2 . It is not possible separation of these radicals. We are not able to strictly define what specific radicals are included in the investigated plant material. We can speculate that these radicals may be derived from the organic compounds of iron, from the various fractions of this iron. They can also originate from other organic compounds in dried plant material. The radicals may also originate from the dried fat. Some of these radicals can be generated by the drying process. These are long-lived radicals. They may be the result of natural physicochemical processes occurring in the dried plant material. In the case of starch we had clearly defined from where our thermally generated radicals come [10]. In the case of our material we do not know that. The problem is that the analyzed dried plant material is heterogeneous in its structure. Previously studied by us, potato starch material was homogeneous which allowed in strict manner to identify the type heat generated radicals. In the study of starch radicals were generated artificially by thermal heating of. In our case we are probably dealing with organic radicals produced during the natural course of physiological processes.

Our study, however, give information on how roasting affects the amount and properties of radicals in the dried plant material that is added to cereal coffee and other food products. They are long-lived radicals, which are not harmful.

We have also attempted EPR research the influence of pressurizing time under pressure of $1000 \mathrm{MPa}(1 \mathrm{GPa})$ on natural radicals in the dried, roasted and unroasted of some of the above plant-herbs materials. Dried herbs subdued to pressurizing under pressure of $1 \pm 0.002 \mathrm{GPa}$ for 6, 60, 300, and $1080 \mathrm{~min}$. However the results are ambiguous and they require more detailed analysis.

\section{Conclusions}

Our research has shown how the roasting process affects the properties of radicals in herbal products added to cereal coffee and other food. The roasting process increases the number of long-lived radicals and may affect their recombination. Long-lived organic radicals included in these plants are not harmful.

Therefore, roasted plant products including these radicals can be added to cereal coffee and other food products improving their flavor and aroma.

\section{References}

[1] E. Korbas, L. Dudarski, P. Bodył, E. Wojtowicz, M. Remiszewski, H. Hoppel, Cereal coffee with herbs, Patent specification PL 218168 B1, UPRP, Polish Patent Office 2013.

[2] R. Zawirska-Wojtasiak, E. Wojtowicz, K. Przygoński, M. Olkowicz, J. Sci. Food Agric. 94, 2118 (2014).

[3] B.A. Goodman, E.C. Pascual, C. Yeretzian, Food Chem. 125, 248 (2011).

[4] C. Yeretzian, E.C. Pascual, B.A. Goodman, Food Chem. 131, 811 (2012).

[5] M. Frangopol, P.T. Frangopol, Ch.L. Trichilo, F.E. Geiger, N. Filipescu, J. Org. Chem. 38, 2355 (1973).

[6] H. Østdal, L.H. Skibsted, H.J. Andersen, Free Radic. Biol. Med. 23, 754 (1997).

[7] A. Haddy, G. Smith, Comp. Biochem. Phys. B 123, 407 (1999).

[8] J. Kumagai, H. Katoh, T. Miyazaki, J. Hidema, T. Kumagai, J. Radiat. Res. 40, 303 (1999).

[9] K. Dyrek, E. Bidzińska, M. Łabanowska, T. Fortuna, I. Przetaczek, S. Pietrzyk, Starch/Stärke 59, 318 (2007).

[10] A. Krupska, A.B. Więckowski, L. Słomińska, L. Jarosławski, R. Zielonka, Carbohyd. Polym. 89, 54 (2012). 
[11] P. Ramos, B. Pilawa, M. Adamski, Ann. Acad. Med. Siles. 68, 28 (2014).

[12] A.M. Maghraby, in: Current Topics in Ionizing Radiation Research, Ed. M. Nenoi, InTechOpen, 2012, p. 649 .

[13] H.M. Peng, B.F. Choules, W.W. Yao, Z. Zhang, R.D. Webster, P.M. Gill, J. Phys. Chem. B 112, 10367 (2008).

[14] S. Koyama, S. Kodama, K. Suzuki, T. Matsumoto, T. Miyazaki, M. Watanabe, Mutat. Res. Fund. Mol. M. 421, 45 (1998).

[15] W.A. Pryor, M. Tamura, D.F. Church, J. Am. Chem. Soc. 106, 5073 (1984).
[16] H. Østdal, H.J. Andersen, M.J. Davies, Arch. Biochem. Biophys. 362, 105 (1999).

[17] B. Dobosz, K. Drzewiecka, A. Waskiewicz, L. Irzykowska, J. Bocianowski, Z. Karolewski, M. Kostecki, Z. Kruczynski, R. Krzyminiewski, Z. Weber, P. Golinski, Appl. Magn. Reson. 41, 19 (2011).

[18] T. Matsumoto, T. Miyazaki, Y. Kosugi, M. Watanabe, Radiat. Phys. Chem. 49, 547 (1997). 